\title{
Impact of intracoronary nicorandil before stent deployment in patients with acute coronary syndrome undergoing percutaneous coronary intervention
}

\author{
XINGLI XU ${ }^{1}$, XIAOLING LIU ${ }^{1}$, LIWEN YU $^{1}$, JING MA $^{1}$, SUFANG YU ${ }^{2}$ and MEI NI ${ }^{1}$ \\ ${ }^{1}$ Key Laboratory of Cardiovascular Remodeling and Function Research, Chinese Ministry of Education, \\ Chinese National Health Commission and Chinese Academy of Medical Sciences, \\ The State and Shandong Province Joint Key Laboratory of Translational Cardiovascular Medicine, \\ Department of Cardiology, Qilu Hospital of Shandong University, Jinan, Shandong 250012; \\ ${ }^{2}$ Department of Neurology, The Fourth People's Hospital, Liaocheng, Shandong 252002, P.R. China
}

Received December 1, 2018; Accepted November 7, 2019

DOI: $10.3892 /$ etm.2019.8219

\begin{abstract}
The present study aimed to clarify the effect of bolus intracoronary nicorandil on inflammatory, oxidative and adherent indicators in patients with acute coronary syndrome (ACS) undergoing percutaneous coronary intervention (PCI). This randomized controlled trial (RCT) was performed to detect the inflammation and oxidative stress in intracoronary blood both before and after PCI. In total, 65 consecutive patients undergoing PCI were classified into a nicorandil therapy group $(n=32)$ or a placebo group $(n=33)$. All procedures were performed at Shandong University Qilu Hospital, China, during the period from
\end{abstract}

Correspondence to: Dr Mei Ni, Key Laboratory of Cardiovascular Remodeling and Function Research, Chinese Ministry of Education, Chinese National Health Commission and Chinese Academy of Medical Sciences, The State and Shandong Province Joint Key Laboratory of Translational Cardiovascular Medicine, Department of Cardiology, Qilu Hospital of Shandong University, 107 West Wenhua Road, Jinan, Shandong 250012, P.R. China

E-mail: nimei@sdu.edu.cn

Abbreviations: ACS, acute coronary syndrome; AHF, acute heart failure; CABG, coronary artery bypass grafting; CAD, coronary artery disease; ELISA, enzyme-linked immunosorbent assay; FFR, fractional flow reserve; IC, intracoronary; MACE, major adverse cardiovascular events; MDA, malondialdehyde; MI, myocardial infarction; NSTEMI, non-ST-elevation myocardial infarction; PCI, percutaneous coronary intervention; PTCA, percutaneous transluminal coronary angioplasty; ROS, reactive oxygen species; sCD40L, soluble CD40 ligand; SOD, superoxide dismutase; STEMI, ST-elevated myocardial infarction; TNF $\alpha$, tumor necrosis factor $\alpha$; hs-CRP, high-sensitivity C-reactive protein; ICAM-1, intercellular adhesion molecule 1; VCAM-1, vascular cell adhesion molecule 1; UA, unstable angina

Key words: nicorandil, anti-inflammation, anti-oxidative stress, acute coronary syndrome, percutaneous coronary intervention
March, 2016 to May, 2017. Intracoronary blood from patients who received nicorandil therapy during PCI showed no change in soluble CD40 ligand (sCD40L) concentration $(1.86 \pm 0.08$ vs. $1.90 \pm 0.09 \mathrm{ng} / \mathrm{ml}, \mathrm{P}=0.12)$ but a significant increase was noted in the control group $(1.87 \pm 0.17$ vs. $2.82 \pm 0.26 \mathrm{ng} / \mathrm{ml}$, $\mathrm{P}<0.01)$. This indicated a relative reduction in $\mathrm{SCD} 40 \mathrm{~L}$ level after PCI in the nicorandil group. We further demonstrated an increase in superoxide dismutase (SOD) activity $(29.37 \pm 0.81$ vs. $31.03 \pm 0.60 \mathrm{U} / \mathrm{ml}, \mathrm{P}<0.001)$ and a reduction in lipid peroxidation ( $3.84 \pm 0.99$ vs. $4.23 \pm 0.13 \mathrm{U} / \mathrm{ml}, \mathrm{P}=0.001)$ in the nicorandil group but observed no change in the placebo group. ICAM-1 levels showed no change in the nicorandil group $(69.54 \pm 6.89$ vs. $72.01 \pm 8.25 \mathrm{ng} / \mathrm{ml}, \mathrm{P}=0.83)$ but a significant increase in the control group after PCI in intracoronary blood $(56.57 \pm 4.96$ vs. $76.81 \pm 6.88 \mathrm{ng} / \mathrm{ml}, \mathrm{P}=0.002)$. No changes were found in hs-CRP, TNF $\alpha$ and SVCAM-1 levels in coronary blood for both groups before and after PCI in ACS patients. Our findings demonstrate that intracoronary bolus nicorandil therapy has a significant effect on the inhibition of inflammatory indicators and oxidative stress in patients with ACS during PCI. This suggests a possible medical application of nicorandil for reducing inflammation and oxidative stress.

\section{Introduction}

Percutaneous coronary interventions (PCIs) are widely performed as an invasive intervention to treat patients with acute coronary syndromes (ACSs) including non-ST-elevation ACS [including unstable angina (UA) and non-ST-elevation myocardial infarction (NSTEMI)] and ST-elevated myocardial infarction (STEMI). It is well-established that PCI induces elevated levels of inflammatory mediators and pathophysiological levels of reactive oxygen species (ROS) production, which leads to an occurrence of restenosis, stent thrombosis and other adverse cardiovascular events in patients with ACS after PCI (1-4).

Inflammatory markers have been implicated as the most important risk indicators in the development of restenosis and may be predictive of poor cardiovascular outcomes in ACS 
patients undergoing coronary angiography (4-7). The concentration of inflammatory cytokines also correlates with the occurrence of stent restenosis. Furthermore, oxidative stress is involved in the pathogenesis of cardiovascular diseases, which is characterized by an imbalance between the generation of ROS and the capacity of the intrinsic antioxidant defense system (8). Research has documented that oxidative stress indicators released from stenotic and symptomatic lesions in patients undergoing PCI participate in the development of new lesions, and increase the risk of major adverse cardiovascular events (MACE) such as cardiovascular death, myocardial infarction (MI) and stroke (9-13).

Nicorandil is a hybrid agent with distinctive pharmacological function as an ATP-sensitive potassium channel $\left(\mathrm{K}_{\mathrm{ATP}}\right)$ opener and a nitric oxide donor. The mechanism of nicorandil includes not only dilation of both the macrovascular and microvascular systems but also has potential cardio-protective effects. Intracoronary (IC) injection of nicorandil has been reported to be safe for patients with coronary artery disease (CAD) during PCI (14-18). Additionally, it was found to improve the prognosis of patients with acute heart failure (AHF) (16), and to ameliorate both early functional defection (17) and long-term complications of acute myocardial infarction (AMI). However, the effects of intracoronary nicorandil injection on inflammation and oxidative stress remain unclear in patients with ACS undergoing PCI as no data are available at present. Thus, the present study was performed to evaluate the feasibility and efficacy of IC nicorandil injection for the improvement of inflammation and oxidative stress in patients with ACS undergoing PCI.

\section{Materials and methods}

Study population. This single-blinded, randomized prospective and clinical trial was performed at the Department of Cardiology, Qilu Hospital, Shandong University, Jinan, Shandong, China. Patients were eligible if they were age 30 years or older and admitted to our institution for ACS. The diagnosis of ACS was based on clinical symptoms, electrocardiographic changes compatible with AMI, and elevation of cardiac biomarkers (18). This study enrolled patients with AMI and unstable angina pectoris. Clinical symptoms of ACS were as follows: Angina pectoris including chest pain with or without radiating to the neck, jaw, upper abdomen or shoulder. AMI was defined as episodes of chest pain persisting $>30$ min and $<24 \mathrm{~h}$, ST-segment elevation in at least two continuous electrocardiogram leads, and more than two-fold creatine kinase (CK) elevation above the maximum peak in the normal range (19). The diagnosis of unstable angina pectoris was based on typical precordial chest pain at rest, angiographic evidence of a stenosis $\geq 75 \%$ according to the American Heart Association (AHA) classification (11) and no elevation of cardiac biomarkers.

Exclusion criteria were as follows: Age $>90$ years; patients with history of coronary artery bypass grafting (CABG); systolic blood pressure (BP), $80 \mathrm{mmHg}$; known allergy to aspirin, clopidogrel, ticagrelor or nicorandil; bleeding history within the prior 3 months; cancer or other severe comorbidity affecting life expectancy. NSTEMI patients are not eligible for stent implantation, thus they were also excluded.
Finally, 65 consecutive participants with a diagnosis of ACS were recruited to the trial after providing written informed consent for angiography, PCI and blood extraction. All patients were admitted to our hospital between March 2016 and May 2017. This study was approved by the Qilu Hospital Ethics Committee (KYLL-2015266). All procedures were performed as standard interventional techniques following current guidelines at the time of intervention.

Procedures. Sixty-five patients with ACS were randomly divided into two groups: A nicorandil treatment group and a control group. The 32 patients in the nicorandil group received an IC bolus of nicorandil $(2 \mathrm{mg} / 2 \mathrm{ml})$ before reperfusion with $\mathrm{PCI}$ and the 32 patients in the control group received the same volume of physiological saline. PCIs were performed due to stenosis-related coronary artery. Next, 3,000 units of heparin was injected after the achievement of arterial access, and adjunctive injections of 7,000 units were needed for patients undergoing PCI. IC administration of nitroglycerin was used to achieve the maximal vasodilation for all patients before the initial and final angiograms. PCI was performed following standard procedures.

A total dose of $12 \mathrm{mg}$ nicorandil was dissolved in $12 \mathrm{ml}$ of $0.9 \%$ saline. Two milliliters of nicorandil in the nicorandil group and $2 \mathrm{ml}$ saline in the control group were administered, respectively, by IC bolus injection before percutaneous transluminal coronary angioplasty (PTCA). All other procedures were the same for both treatment groups. The cardiologist in-charge performed PCI and IC injection of nicorandil or saline in the catheter laboratory at the appropriate time. No angiographical residual stenosis was detected in any patients and there were no deaths during PCI. No follow-up data, such as complications, mortality and long-term effect of nicorandil, were available in this study. After all procedures, patients were maintained on aspirin (100 mg once daily) plus clopidogrel (75 mg once daily) or ticagrelor (90 mg twice daily) for at least 1 year after metal stent placement followed by the physician's specific directions.

Collection and preservation of coronary blood. Approximately $10 \mathrm{ml}$ of intracoronary blood was collected from the distal coronary stenosis with a Finecross micro-catheter (Asahi Intecc Co., Ltd.) before the injection of nicorandil or saline prior to PTCA, and immediately after the stent placement in patients undergoing PCI. The blood samples were collected in vacutainer anticoagulant tubes at room temperature for $\sim 30 \mathrm{~min}$ and then centrifuged for $10 \mathrm{~min}$ at $1,000 \mathrm{x}$ g. Plasma and serum were aliquoted and stored at $-80^{\circ} \mathrm{C}$ until further analysis.

Measurement of SOD and MDA. Superoxide dismutase (SOD) was examined using an SOD determination kit (cat. no. 19160; Sigma-Aldrich; Merck KGaA) following the manufacturer's instructions. Each sample was measured with three replicates of four samples: Sample, Blank 1, Blank 2 and Blank 3. The Sample subset consisted of $20 \mu \mathrm{l}$ sample solution, $200 \mu \mathrm{l}$ of WST working solution and $20 \mu$ l of enzyme working solution. Then the sample solution was substituted by $20 \mu 1$ of enzyme working solution as Blank 1. Blank 2 consisted of $20 \mu \mathrm{l}$ sample solution, $200 \mu \mathrm{l}$ of WST working solution and $20 \mu \mathrm{l}$ of dilution 
buffer. Finally, $20 \mu \mathrm{l}$ sample solution was substituted by $20 \mu \mathrm{l}$ of enzyme working solution in Blank 3 compared with Blank 2. The plates were incubated at $37^{\circ} \mathrm{C}$ for $20 \mathrm{~min}$ and the absorbance was read at $450 \mathrm{~nm}$ using a microplate reader. The SOD activity calculation formula is as follows: SOD activity (inhibition rate $\%)=\left\{\left(\left(\mathrm{A}_{\text {Blank 1 }}-\mathrm{A}_{\text {Blank } 3}\right)-\left(\mathrm{A}_{\text {Sample }}-\mathrm{A}_{\text {Blank } 2}\right)\right) /\left(\mathrm{A}_{\text {Blank } 1^{-}}\right.\right.$ A $\left.\left._{\text {Blank3 }}\right)\right\}$ x100.

Malondialdehyde (MDA) was measured with the Lipid Peroxidation Assay Kit (cat. no. MAK085; Sigma-Aldrich; Merck $\mathrm{KGaA}$ ) according to the manufacturer's instructions. The MDA standard solution was used to generate blank, $0.4,0.8,1.2,1.6$ and $2.0 \mathrm{nmol}$ standards to create a standard curve. Plasma samples $(10 \mu \mathrm{l})$ were gently mixed with $500 \mu \mathrm{l}$ of $42 \mathrm{mM}$ sulfuric acid. Then $125 \mu \mathrm{l}$ of phosphotungstic acid solution was added and mixed by vortexing. After incubation at room temperature for $5 \mathrm{~min}$ and centrifugation at $13,000 \mathrm{x} \mathrm{g}$ for $3 \mathrm{~min}$, the pellet was resuspended on ice with a water/BHT solution. TBA solution was added to each sample including standards and they were incubated at $95^{\circ} \mathrm{C}$ for $1 \mathrm{~h}$. Then, $200 \mu \mathrm{l}$ of each sample mixture and standard were pipetted into a 96-well plate and the absorbance was measured at $532 \mathrm{~nm}$. The concentrations of MDA in samples were calculated using the previously determined standard curve. Three replicates were conducted for each sample.

Measurement of $s C D 40 L$, tumor necrosis factor $\alpha$ (TNF $\alpha)$, high-sensitivity $C$-reactive protein ( $h s-C R P)$, intercellular adhesion molecule-1 (ICAM-1) and vascular cell adhesion molecule-1 (VCAM-1). Enzyme-linked immunosorbent assay (ELISA) was used to determine the inflammatory factors for each plasma sample according to the manufacturer's instructions. A serial dilution of purified standards was used to generate a standard curve to measure the cytokine levels in a semi-quantitative way. Kits purchased from R\&D Systems were used to detect the plasma or serum levels of soluble CD40L (cat. no. DCDL40, Human CD40 Ligand/TNFSF5 Quantikine ELISA kit), TNF $\alpha$ (cat. no. DTA00D, Human TNF- $\alpha$ Quantikine ELISA kit), hs-CRP (cat. no. DCRP00, Human C-Reactive Protein/CRP Quantikine ELISA kit), I-CAM (cat. no. DCD540, Human ICAM-1/CD54 Allele-specific Quantikine ELISA kit) and V-CAM (Cat. no. PDVC00, Human sVCAM-1/CD106 Quantikine ELISA kit). Three replicates were examined for each sample.

Statistical analysis. Data are shown as the mean \pm SD and three replicates were conducted for each analysis. Differences between the nicorandil and placebo groups were analyzed by the two-tailed paired t-test for continuous outcome variables. This test was used for comparing the pre-PCI and post-PCI values within the nicorandil and control groups. The comparisons between non-continuous variables were performed using the $\chi^{2}$ test. SPSS statistical software package, version 17.0 (SPSS Inc.) was used for analysis. A P-value $<0.05$ was considered statistically significant.

\section{Results}

Patient baseline characteristics. In this study, a total of 65 consecutive patients were enrolled with 32 randomly allocated to the nicorandil and 33 to placebo groups. The baseline characteristics of the two groups are shown in Table I. There were no significant differences in age, sex, BMI, incidence of coronary risk factors, serum creatinine or urea nitrogen levels, ejection fraction (EF), previous MI history, culprit lesions or quantitative angiographic data between the two groups. Clinically, 5 patients $(15.6 \%)$ and 3 patients $(9.1 \%)$ were diagnosed with myocardial infarction (MI) in the nicorandil group and placebo group, respectively. Other patients were diagnosed with unstable angina (UA). There were no significant differences in the use of oral medications at discharge among all patients.

Outline of the PCI procedures. Coronary angiography was performed to visualize the severity of coronary stenosis. The subsequent treatment of PTCA was followed up if the residual diameter stenosis was greater than or equal to $75 \%$ of the coronary artery stenosis diameter. The Finecross micro-catheter reached the distal coronary stenosis guided by a coronary guide-wire, and $10 \mathrm{ml}$ of coronary blood was collected before PCI. Then $2 \mathrm{ml}$ nicorandil $(1 \mathrm{mg} / \mathrm{ml})$ or placebo ( $2 \mathrm{ml}$ of $0.9 \%$ saline) was IC bolus injected into coronary ostia. After nicorandil (or placebo) injection, PCI was performed according to standard techniques. Another $10 \mathrm{ml}$ of coronary blood was collected immediately after stent implantation (Figs. 1 and S1).

Effect of nicorandil on markers of inflammation. There was a significant increase in soluble CD40 ligand (sCD40L) levels in the plasma of post-PCI compared with pre-PCI in the control group ( $1.87 \pm 0.17$ vs. $2.82 \pm 0.26 \mathrm{ng} / \mathrm{ml}, \mathrm{P}<0.001)$, but no difference in the nicorandil group in both pre- and post-PCI (1.86 \pm 0.08 vs. $1.90 \pm 0.09 \mathrm{ng} / \mathrm{ml}, \mathrm{P}=0.12)$ (Fig. $2 \mathrm{~A}$ ). Compared with post-PCI in the control group, there was a significant reduction in SCD40L levels in the nicorandil group $(\mathrm{P}=0.001)$ (data not shown). The amount of change ( $\Delta$ value) for $\mathrm{sCD} 40 \mathrm{~L}$ was significantly increased in the control group with no difference in the nicorandil group $(0.04 \pm 0.04$ vs. $0.95 \pm 0.23 \mathrm{ng} / \mathrm{ml}, \mathrm{P}<0.001$; Fig. 2A). No significant change was found in pre- and post-PCI levels of TNF $\alpha$ in the nicorandil group $(5.90 \pm 0.84$ vs. $5.3 \pm 2.73 \mathrm{pg} / \mathrm{ml}, \mathrm{P}=0.43)$ and control group $(6.13 \pm 0.58$ vs. $7.08 \pm 0.95 \mathrm{pg} / \mathrm{ml}, \mathrm{P}=0.29)$ (Fig. 2B). In comparison, there was no difference between pre-PCI and post-PCI in levels of IC plasma hs-CRP in the nicorandil group $(5.16 \pm 1.05$ vs. $6.13 \pm 1.13 \mathrm{mg} / \mathrm{ml}, \mathrm{P}=0.054)$ and in the control group $(5.98 \pm 1.01$ vs. $6.64 \pm 1.12 \mathrm{mg} / \mathrm{ml}$, $\mathrm{P}=0.458$ ) (Fig. 2C). The $\Delta$ value of both TNF $\alpha(-0.60 \pm 0.75$ vs. $0.95 \pm 0.84 \mathrm{pg} / \mathrm{ml}, \mathrm{P}=0.405)$ and hs-CRP $(0.98 \pm 0.49$ vs. $0.66 \pm 0.88 \mathrm{mg} / \mathrm{ml}, \mathrm{P}=0.246)$ remained statistically unchanged (Fig. 2B and C). Thus, the inflammatory sCD40L marker was significantly improved during PCI due to the intracoronary bolus injection of nicorandil.

Effect of nicorandil on indicators of oxidative stress. SOD and MDA indicators were measured to investigate the effect of nicorandil on oxidative stress. SOD was significantly increased in the plasma of post-PCI compared with pre-PCI in nicorandil group $(29.37 \pm 0.81$ vs. $31.03 \pm 0.60 \mathrm{U} / \mathrm{ml}, \mathrm{P}<0.001)$, and no difference was observed between time-points in the control group $(29.29 \pm 0.68$ vs. $29.58 \pm 0.73 \mathrm{U} / \mathrm{ml}, \mathrm{P}=0.519)$ (Fig. 3A). Additionally, MDA was significantly increased in 
Table I. Patients and characteristics of the lesion.

\begin{tabular}{|c|c|c|c|}
\hline \multirow[b]{2}{*}{ Characteristics } & \multicolumn{3}{|c|}{ Groups } \\
\hline & Nicorandil $(n=32)$ & Placebo $(n=33)$ & P-value \\
\hline Age, in years & $60.0 \pm 10.0$ & $61.8 \pm 9.7$ & 0.466 \\
\hline Male, n (\%) & $21(65.6)$ & $21(63.6)$ & 0.869 \\
\hline $\mathrm{BMI}, \mathrm{kg} / \mathrm{m}^{2}$ & $26.7 \pm 4.1$ & $26.0 \pm 3.3$ & 0.459 \\
\hline \multicolumn{4}{|l|}{ Cardiovascular risk factors, $\mathrm{n}(\%)$} \\
\hline Hypertension & $23(71.9)$ & $22(66.7)$ & 0.655 \\
\hline Diabetes mellitus & $8(25)$ & $11(34.4)$ & 0.468 \\
\hline Hyperlipidemia & $12(37.5)$ & $13(39.4)$ & 0.878 \\
\hline Smoking & $16(50)$ & $15(45.5)$ & 0.719 \\
\hline $\mathrm{Cr}, \mu \mathrm{mol}-/ \mathrm{l}$ & $72.91 \pm 11.63$ & $69.94 \pm 17.75$ & 0.430 \\
\hline $\mathrm{BUN}, \mathrm{mg} / \mathrm{dl}$ & $5.22 \pm 1.88$ & $8.89 \pm 15.93$ & 0.198 \\
\hline Previous MI, n (\%) & $3(9)$ & $3(9)$ & 0.968 \\
\hline Number of vessels occluded & $1.33 \pm 0.58$ & $1.33 \pm 0.58$ & 1.000 \\
\hline $\mathrm{EF}, \%$ & $62.8 \pm 6.4$ & $60.1 \pm 8.7$ & 0.157 \\
\hline \multicolumn{4}{|l|}{ Clinical diagnosis, $\mathrm{n}(\%)$} \\
\hline MI & $5(15.6)$ & $3(9.1)$ & 0.431 \\
\hline UA & $28(84.4)$ & $30(90.9)$ & 0.431 \\
\hline \multicolumn{4}{|l|}{ Quantitative coronary angiography } \\
\hline Percent diameter stenosis, $\%$ & $89.3 \pm 7.1$ & $87.0 \pm 7.5$ & 0.236 \\
\hline \multicolumn{4}{|l|}{ Vessel undergoing PCI, $\mathrm{n}$} \\
\hline Left anterior descending artery & 21 & 19 & 0.681 \\
\hline Left circumflex artery & 3 & 4 & 0.726 \\
\hline Right coronary artery & 8 & 10 & 0.468 \\
\hline Vessel diameter, mm & $23.06 \pm 7.52$ & $26.79 \pm 9.04$ & 0.07 \\
\hline \multicolumn{4}{|l|}{ Therapy, \% } \\
\hline Aspirin & 100 & 100 & 1.000 \\
\hline Clopidogrel & 90.9 & 93.8 & 0.378 \\
\hline ACEI & 93.75 & 93.94 & 0.975 \\
\hline ARB & 6.25 & 6.06 & 0.975 \\
\hline$\beta$-blocker & 100 & 100 & 1.00 \\
\hline Statin & 100 & 100 & 1.00 \\
\hline Glycoprotein IIb/IIIa inhibitor & 9.1 & 6.2 & 0.623 \\
\hline
\end{tabular}

Data are expressed as mean $\pm \mathrm{SD}, \mathrm{n}(\%)$, or median (interquartile range). BMI, body mass index; $\mathrm{Cr}$, creatinine; BUN, blood urea nitrogen; MI, myocardial infarction; EF, ejection fraction; UA, unstable angina; ACEI, angiotensin-converting enzyme inhibitors; ARB, angiotensin receptor blocker; PCI, percutaneous coronary intervention. The t-test or $\chi^{2}$ test was used to assess the significance of the differences between the two groups.

plasma of post-PCI compared to pre-PCI in the nicorandil group ( $3.84 \pm 0.10$ vs. $4.230 \pm 0.13 \mathrm{mmol} / \mathrm{ml}, \mathrm{P}=0.001)$, and no difference was observed between time-points in the control group (3.78 \pm 0.11 vs. $3.85 \pm 0.13 \mathrm{mmol} / \mathrm{ml}, \mathrm{P}=0.402)$ (Fig. 3B). Surprisingly, the ratio of SOD and MDA was unchanged in four time-point subgroups of both the nicorandil $(7.84 \pm 0.33$ vs. $7.58 \pm 0.30, \mathrm{P}=0.241)$ and control $(7.92 \pm 0.27$ vs. $7.95 \pm 0.35$, $\mathrm{P}=0.888$ ) group (Fig. 3C). There also was no difference in the $\Delta$ value of SOD $(1.28 \pm 0.39$ vs. $0.29 \pm 0.45 \mathrm{U} / \mathrm{ml}, \mathrm{P}=0.328)$ in the control vs. nicorandil group, but a significant increase in levels of MDA $(-0.70 \pm 0.26$ vs. $0.07 \pm 0.08 \mathrm{mmol} / \mathrm{ml}$, $\mathrm{P}<0.001)$ in the control group compared with the nicorandil group (Fig. 3A and B).
These results indicated that nicorandil may relieve oxidative stress via increased levels of SOD, but the levels of MDA were also subsequently increased. The possible change in ratio between SOD and MDA during PCI may be based on operational injury and the delayed reaction of the plasma levels to oxidative stress indicators.

Effect of nicorandil on angiokinetic factors. To further explore the effects of bolus injection of nicorandil during PCI, we also measured two different functional angiokinetic factors, ICAM-1 and sVCAM-1 post-PCI compared with pre-PCI. The results showed no significant differences in ICAM-1 in the nicorandil group $(64.79 \pm 3.63$ vs. $63.80 \pm 5.85 \mathrm{ng} / \mathrm{ml}, \mathrm{P}=0.827)$. 

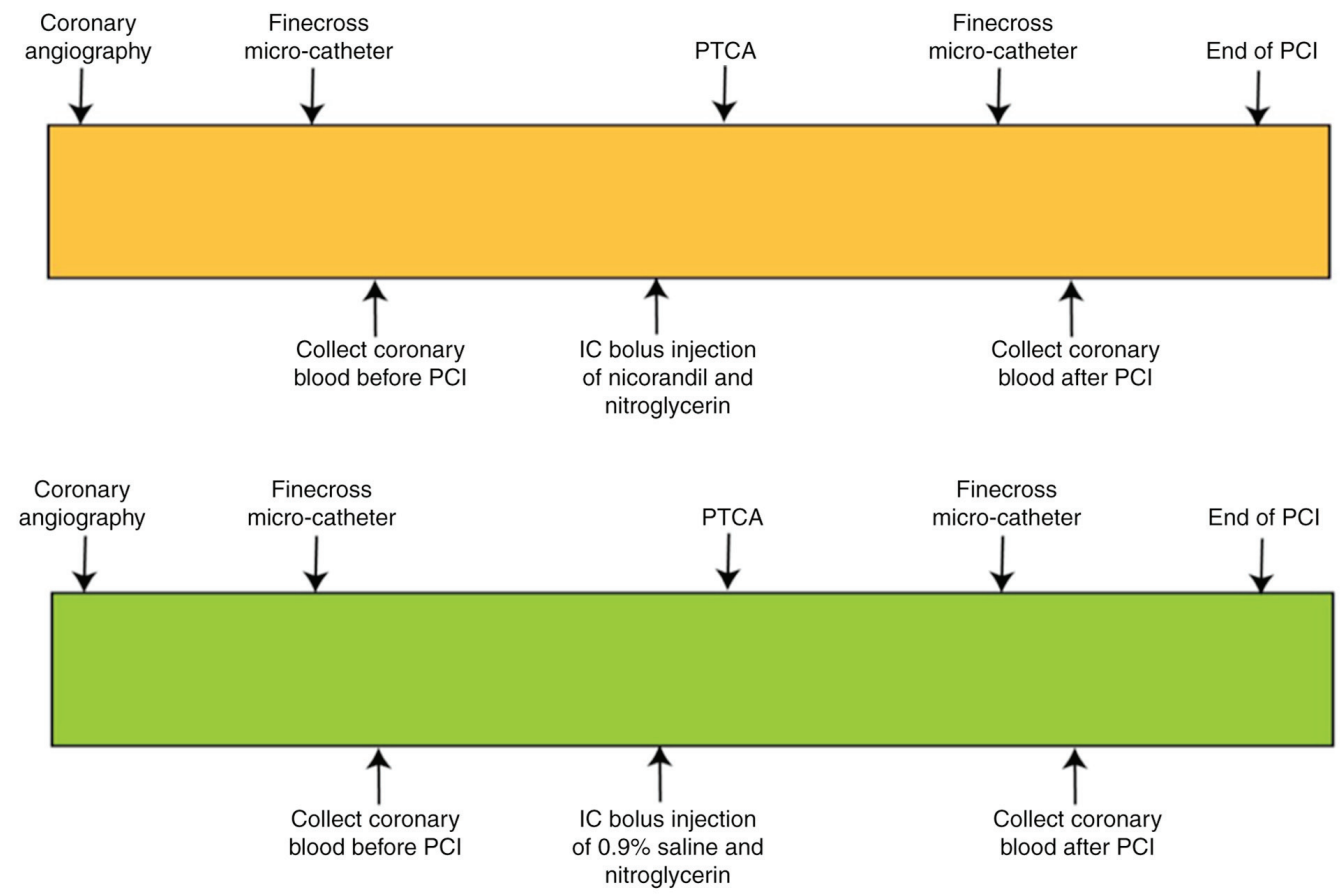

Figure 1. Outline of the study procedures. Patients with UA or STEMI underwent PCI performed according to the standard techniques and using 1 of 2 strategies: i) PCI with nicorandil IC bolus injection before PTCA or ii) PCI followed by the same volume of $0.9 \%$ saline IC bolus injection before PTCA. PCI, percutaneous coronary intervention; PTCA, percutaneous transluminal coronary angioplasty; UA, unstable angina; STEMI, ST-elevated myocardial infarction.
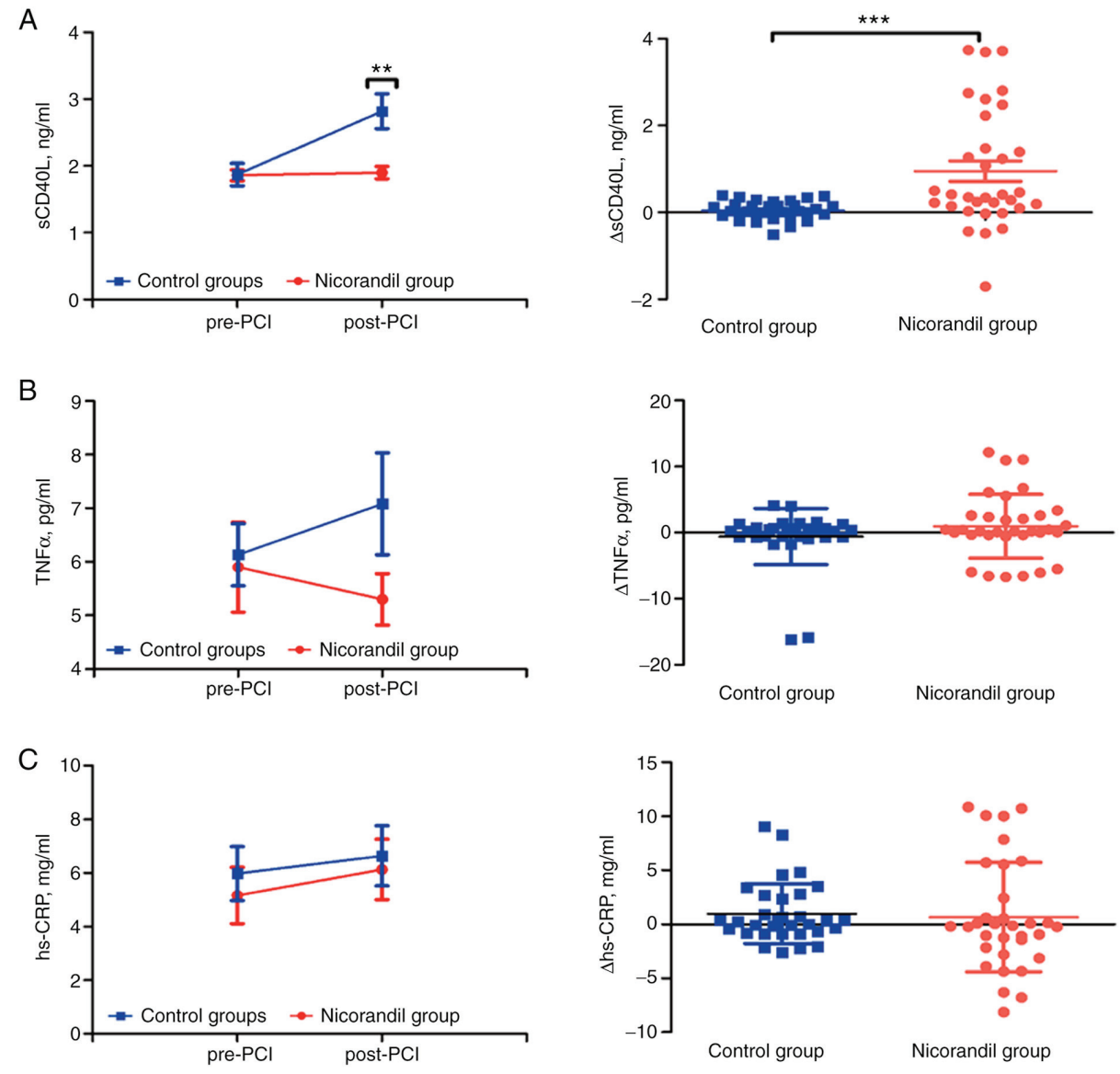

Figure 2. Dynamics of the inflammatory response pre-PCI and post-PCI, and the difference in values between the two subgroups in the nicorandil and control groups. (A) Levels of sCD40L before and after PCI in the groups and subgroups, and $\Delta$ values of sCD40L of post-PCI compared with pre-PCI in the two groups. (B) Levels of TNF $\alpha$ before and after PCI in the groups and subgroups, and $\Delta$ values of TNFa of post-PCI compared with pre-PCI in the two groups. (C) Levels of hs-CRP before and after PCI in the groups and subgroups, and different values of hs-CRP of post-PCI compared with pre-PCI in two groups. Values are expressed as mean $\pm \mathrm{SD} ; \Delta$ values are expressed as mean $\pm \mathrm{SD} ;{ }^{* * *} \mathrm{P}<0.01,{ }^{* * * *} \mathrm{P}<0.001$. PCI, percutaneous coronary intervention; sCD40L, soluble $\mathrm{CD} 40$ ligand; $\mathrm{TNF} \alpha$, tumor necrosis factor $\alpha$; hs-CRP, high-sensitivity C-reactive protein. 

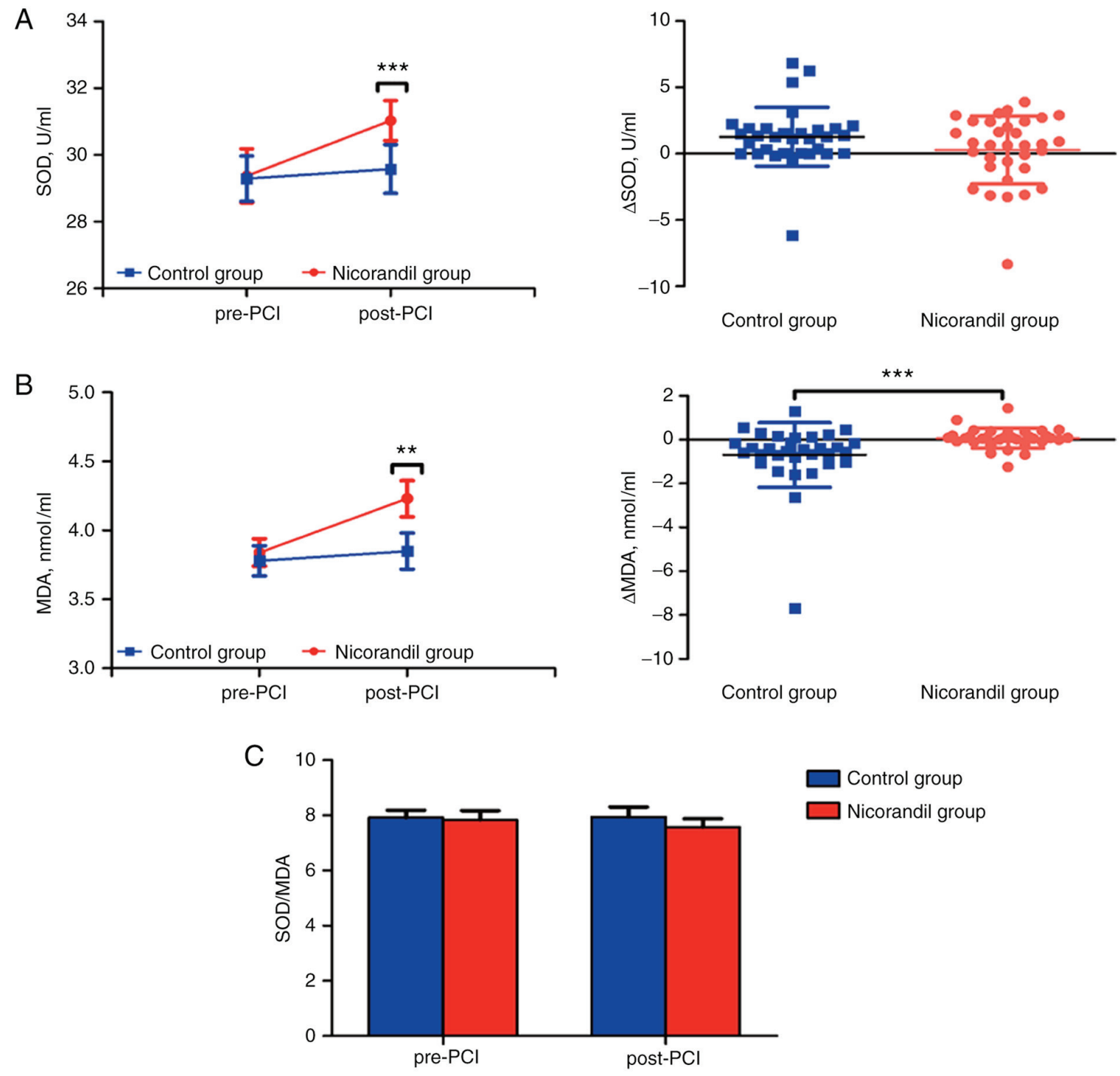

Figure 3. Dynamics of the oxidative stress in pre-PCI and post-PCI subgroups, and the difference in values between the two subgroups in the nicorandil and control groups. (A) Levels of SOD before and after PCI in the groups and subgroups, and $\Delta$ values of SOD of post-PCI compared with pre-PCI in the two groups. (B) Levels of MDA before and after PCI in the groups and subgroups, and $\Delta$ values of MDA of post-PCI compared with pre-PCI in the two groups. (C) Ratio of SOD and MDA in the subgroups and nicorandil and control groups. Values are expressed as mean \pm SD; $\Delta$ values are expressed as mean \pm SD; ${ }^{* *} \mathrm{P}<0.01,{ }^{* * *} \mathrm{P}<0.001$. PCI, percutaneous coronary intervention; MDA, malondialdehyde; SOD, superoxide dismutase.

However, levels of ICAM-1 in the control group were increased after PCI $(66.30 \pm 4.17$ vs. $75.24 \pm 5.46 \mathrm{ng} / \mathrm{ml}, \mathrm{P}=0.002)$ (Fig. 4A). There was also no change in levels of sVCAM-1 in the nicorandil group $(4.85 \pm 0.61 \mathrm{vs} .5 .48 \pm 0.61 \mu \mathrm{g} / \mathrm{ml}, \mathrm{P}=0.175)$ compared to placebo group $(5.13 \pm 0.46$ vs. $5.46 \pm 0.58 \mu \mathrm{g} / \mathrm{ml}$, $\mathrm{P}=0.255)$ (Fig. 4B). The $\Delta$ values both for ICAM-1 (-0.99 \pm 6.12 vs. $8.94 \pm 4.10 \mathrm{ng} / \mathrm{ml}, \mathrm{P}=0.285)$ and sVCAM-1 $(-0.63 \pm 0.46$ vs. $-0.33 \pm 0.28 \mu \mathrm{g} / \mathrm{ml}, \mathrm{P}=0.927)$ also showed no difference between the two groups (Fig. 4A and B). Nicorandil may improve the angiokinetic factor of ICAM-1 to hinder any inflammatory reaction during PCI in ACS patients.

\section{Discussion}

The present study presents for the first time the effects of intracoronary (IC) bolus injection of nicorandil or placebo on markers of inflammation and oxidative stress in patients with acute coronary syndrome (ACS) undergoing percutaneous coronary interventions (PCIs). In this randomized, single-blinded and placebo-controlled study, it was confirmed in a brief period that percutaneous transluminal coronary angioplasty (PTCA) results in the upregulation of inflammation and oxidative stress. Coronary blood, which has a greater impact on the coronary artery compared with the peripheral blood, was used to evaluate serum as well as plasma levels of inflammatory and oxidative markers to precisely investigate temporal changes during PCI. It was also demonstrated that an IC bolus injection of nicorandil in patients with ACS is safe and significantly reduced soluble CD40 ligand (sCD40L), superoxide dismutase (SOD), malondialdehyde (MDA) and intercellular adhesion molecule-1 (ICAM-1) levels after PCI. However, no changes in other systemic inflammation markers, such as high-sensitivity C-reactive protein (hs-CRP), tumor necrosis factor $\alpha$ (TNF $\alpha$ ) and soluble vascular cell adhesion molecule-1 (sVCAM-1) levels were observed in the nicorandil or control groups. This finding could possibly be due to delayed inflammatory responses. 

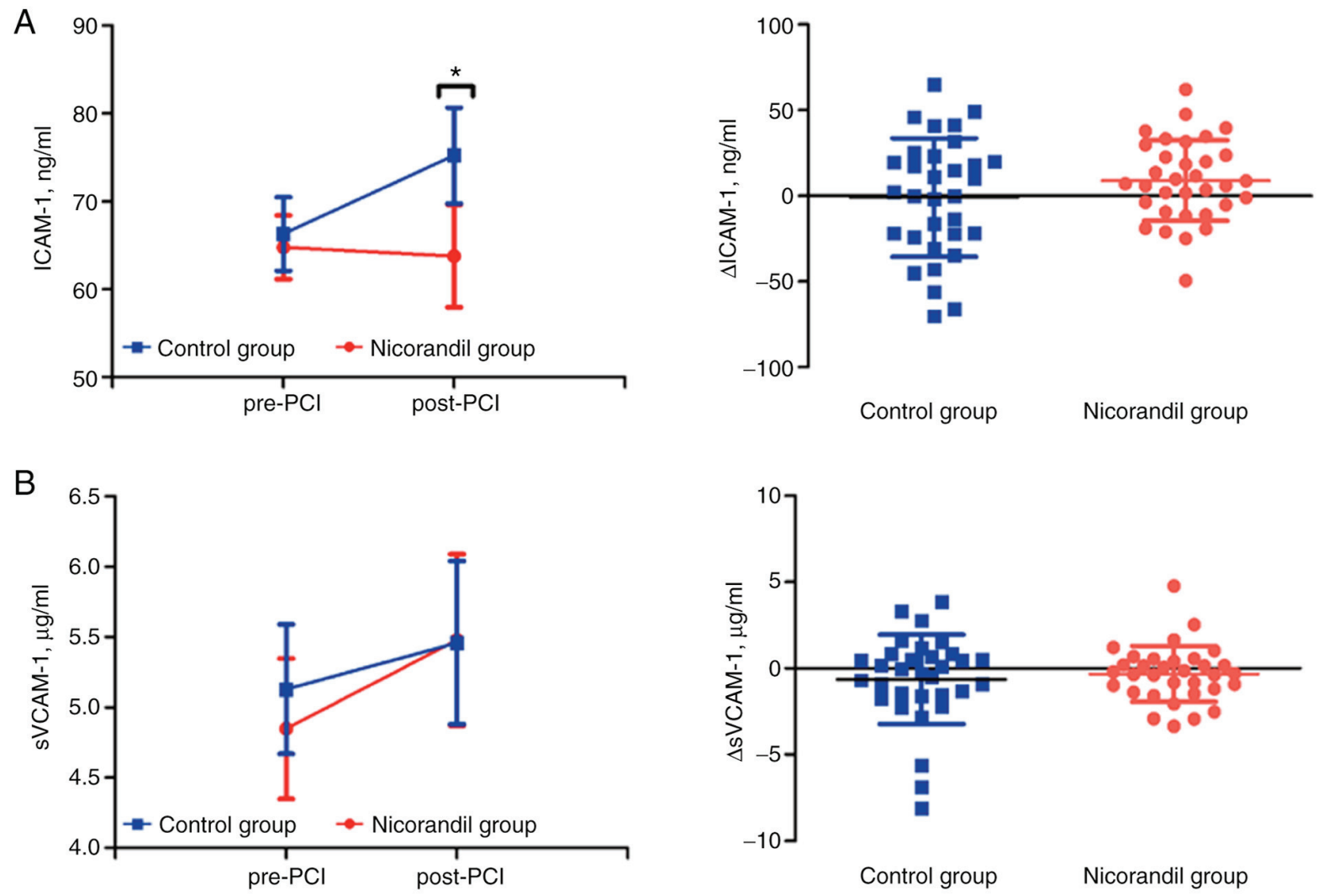

Figure 4. Dynamics of the angiokinetic factors in pre-PCI and post-PCI subgroups, and the difference in values between the two subgroups in the nicorandil and control groups. (A) Levels of ICAM-1 before and after PCI in the groups and subgroups, and $\Delta$ values of ICAM-1 of post-PCI compared with pre-PCI in two groups. (B) Levels of sVCAM-1 before and after PCI in the groups and subgroups, and $\Delta$ values of sVCAM-1 of post-PCI compared with pre-PCI in the two groups. Values are expressed as mean $\pm \mathrm{SD} ; \Delta$ values are expressed as mean $\pm \mathrm{SD}$; "P<0.05. PCI, percutaneous coronary intervention; ICAM-1, intercellular adhesion molecule-1; VCAM-1, vascular cell adhesion molecule-1.

PCI has become a standard revascularization procedure and the main management option for patients with ACS. However, PTCA induces vascular injury and wreaks damage on the endothelium which is associated with pro-inflammatory responses and platelet-activating effects with adhesion $(20,21)$. This damage also elicits an inflammatory response via the activation of platelets and leukocytes (22). PCI-induced vascular injury and ischemia/reperfusion injury was found to result from both intracellular calcium loading due to ATP depletion and intracellular acidification (23), and the occurrence of oxidative stress with the mitochondrial respiratory chain as the main source of reactive oxygen species (ROS) (24).

Nicorandil, as a hybrid nitrate and ATP-sensitive potassium channel opener agent (25), has been shown not only to dilate systemic veins, but also to exert a vaso-dilative effect on arteries, including peripheral arteries (16). Moreover, IC bolus injection of nicorandil is safe and efficacious for myocardial protection, ameliorating fractional flow reserve (FFR) and ischemia/reperfusion injury $(26,27)$. Kawai et al found that intravenous administration of nicorandil could prevent slow coronary flow phenomenon compared with that of $0.9 \%$ saline before PCI in patients with ACS or non-ACS (28). Previous research further found that IC bolus injection of nicorandil is safe and effective compared with IC adenosine to induce hyperemia in patients with an angiographically intermediate lesion (27). Lee et al compared IC bolus injection of nicorandil with intravenous infusion of adenosine (26). Different from previous studies, we firstly IC injected the same volume of saline in ACS patients since no placebo effect was observed in those studies which further increased the importance of our study. We also collected coronary blood to perform ELISA to evaluate the levels of inflammatory or oxidative indicators during PCI. A previous study also showed that nicorandil improves microvascular dysfunction as assessed by the index of microcirculatory resistance after PCI (29).

The soluble CD40 ligand (sCD40L), as a member of the TNF family, is expressed widely on monocytes, dendritic cells, endothelial cells and epithelial cells (30). Activation of platelets was shown to result in the release of SCD40L, accelerating the inflammatory process and promoting coagulation (31-34). A previous study showed that $\mathrm{SCD} 40 \mathrm{~L}$ may be a predictor of coronary artery disease in patients undergoing PCI (35). Thus, we evaluated the level of SCD40L in pre-PCI and post-PCI subgroups in the nicorandil and control groups. Its expression level in intracoronary plasma increased after the stent implantation compared with pre-PCI in the placebo group which is consistent with other trials $(35,36)$. The IC bolus injection of nicorandil also improved the alleviation of $\mathrm{SCD} 40 \mathrm{~L}$ in the post-PCI subgroup immediately as there were no significant changes compared with the post-PCI and pre-PCI subgroup. Our trial further confirmed that nicorandil has the ability to improve the inflammatory reaction of sCD40L during PCI in ACS patients. 
Other inflammation indicators, such as TNF $\alpha$ and hs-CRP, had no significant differences in both nicorandil and placebo groups and their subgroups. Although PCI led to a platelet-activating effect with adhesion and sCD40L participates in the promotion of coagulation, the adhesion molecule sVCAM-1 also exhibited no difference in the different groups although ICAM-1 was clearly induced in the control group after PCI. The reasons for this finding may be as follows. Firstly, Aströmolsson et al demonstrated that TNF $\alpha$ increased during the $24 \mathrm{~h}$ of reperfusion (37). Hs-CRP, an acute-phase reactant, increased markedly at $6 \mathrm{~h}$ after PCI and reached a peak value at $48 \mathrm{~h}$ which indicates that there is a delayed reaction of these inflammatory markers after PCI $(37,38)$. Consequently, the underlying improvement of inflammatory indicators with nicorandil may not react and reflect changes immediately after operation. Secondly, Bayata et al measured VCAM-1 and sICAM-1 serum levels in patients before and after stent implantation and found no difference in VCAM-1 and ICAM-1 levels (39). Similarly, Wexberg et al found increased levels of VCAM-1 after stent implantation (40). Thus, the differences between the above trials and our results may be due to different sourcing of serum or plasma as our samples came from the intracoronary blood drawn by the micro-catheter. Another reason may be a difference in patient ethnicity and the reactivity to PTCA. The small sample size may also be one of the reasons for this difference.

Reactive oxygen species (ROS) are involved in physiological cell regulation and redox signaling (41). SOD, which catalyzes the metabolism of the superoxide anion $\left(\mathrm{O}_{2}^{-}\right)$into hydrogen peroxide and molecular oxygen, is one of the most important antioxidative enzymes. MDA, the major end product of lipid peroxidation, is one of the markers indicating partial levels of oxidative stress. The production of MDA is initiated promptly after the generation of ROS (42). Both molecules directly reflect the expression levels of oxygen-free radicals. Our results showed that both SOD and MDA levels have the tendency to rise but there were no significant differences in both subgroups of the control group. An investigation by Ekeløf et al indicated that SOD was reduced significantly at $2 \mathrm{~h}$ after PTCA (42), which suggests that the consumption of SOD may require a series of processes. However, a study by Demircan et al indicated that SOD was immediately reduced after PCI compared to the pre-PCI subgroup (43). These controversial results require further investigation due to the limited sample sizes employed.

In the present study, it was demonstrated that the SOD levels in the post-PCI subgroup of the nicorandil group significantly increased compared with the pre-PCI subgroup. However, the MDA levels were also unexpectedly increased. The ratio of SOD and MDA exhibited no significant change in the post-PCI nicorandil group compared with the control groups. These results showed that nicorandil reduced oxidative stress significantly as a supplement to PCI. The over-production of free radicals at the beginning of the ischemic phase is thought to be due to mitochondrial depolarization. Nicorandil reduces ROS formation by mitochondria by opening the mitochondrial $\mathrm{K}_{\mathrm{ATP}}(44)$. Due to its free radical scavenging properties and inhibition of oxidative respiratory bursting of neutrophils (45), IC bolus injection of nicorandil could function by reducing oxidative stress. Furthermore, nicorandil plays a role in ameliorating myocardial damage as well as improving cardiac function and clinical outcomes.
The inflammatory response evoked by vascular damage during angioplasty and the subsequent reaction are thought to be the main contributors to the development of restenosis. Oxidative stress also contributes to clinical outcomes such as adverse cardiac events. IC bolus injection of nicorandil before implantation of the coronary artery stent was associated with better suppression of various inflammatory cytokines, and further reduced the oxidative stress in patients who suffered from ACS undergoing PCI. However, there were no definite clinical benefits detected in the amelioration of adhesion molecules and inflammatory mediators. Our findings suggest that IC bolus injection of nicorandil is a simple, safe, and effective way to reduce the levels of inflammation and oxidation during PCI which may be attributable to improved outcomes for such ACS patients.

There were several limitations to the present study. Firstly, the sample size of this study was small. It was necessary to perform a power analysis before conducting the study. However, no similar data could be found in the published articles which made it difficult for us to assess the sample size. Thus, we enrolled 65 patients to ensure the normality of the data distribution. We surprisingly found significant differences of SCD40L, SOD, MDA and ICAM-1 between the two groups. Then, we performed the power analysis through PASS based on our expression levels of sCD40L, SOD, MDA and ICAM-1. We found that the value of $1-\beta$ was beyond 0.8 . Secondly, since nicorandil was only used during PCI and only 20 of the enrolled patients took nicorandil regularly post-discharge, no long-term observations of nicorandil was obtained. We followed up the enrolled patients for only one month after PCI. No difference in complications and mortality was found in the two groups after one month. After surgery, the patients recovered well after regular intake of the standard medicine. Finally, according to the new guidelines of ECS for ACS patients, ticagrelor is recommended for Class IA. However, ticagrelor is recommended for Class IB in China at present. According to the 2016 Ticagrelor Expert Consensus in China (46), ticagrelor should be used as early as possible in NSTE-ACS patients who have a moderate or high risk of ischemia and plan for early invasive treatment. We previously used clopidogrel more often during our experimental period according to our previous guidelines. More than $50 \%$ patients now use ticagrelor instead of clopidogrel at present. We still need to consider our clinical practice, Chinese national conditions, individual differences and implement specific antithrombotic strategies to choose the antiplatelet drugs. We will also follow up the patients taking nicorandil after PCI for a longer period to explore its long-term effect when we carry out a larger sample study in future studies.

In conclusion, our trial showed that IC bolus injection of nicorandil as part of therapy for patients with ACS undergoing PCI reduced soluble CD40 ligand levels, increased MDA and SOD levels, and decreased ICAM-1. However, none of the other subclinical markers of inflammation were altered after the injection of nicorandil and PCI surgery. These findings within the context of this clinical trial suggest a solitary anti-oxidative and anti-inflammatory effect of nicorandil. A larger sample size and a longer treatment period with nicorandil are needed for further investigation of the underlying and long-term beneficial effects of this therapy. 
The prospective, randomized design and use of coronary angiography to diagnose ACS patients are the strengths of this study. The percutaneous transluminal coronary angioplasty (PTCA) showed that a residual stenosis diameter $\geq 75 \%$ of the coronary artery combined with clinical symptoms are the gold standard for identifying ACS patients.

Coronary blood was used to test serum or plasma levels of inflammatory and oxidative markers, which was a better measure of the coronary artery compared with peripheral blood as it precisely reflected the effect on markers after stent deployment.

The limitations of this study included a limited sample size of 65 subjects, inflammatory or oxidative indicators influenced by PTCA and limited time period for extraction of coronary blood during PCA and stent deployment.

\section{Acknowledgements}

This study was facilitated with the assistance of many colleagues in the Department of Cardiology and Cardiac Intervention Department of Shandong University Qilu Hospital. In addition to the dedicated workers, the authors would especially like to thank Professor Gui-Peng An, Wen-Qiang Chen and Qing Zhu who helped in collecting and analyses of the blood samples. We also thank the Professor Lu Wang for his help in English writing. Trial registration: This study was approved by the Qilu Hospital Ethics Committee (KYLL-2015266). Trial registration: Current Controlled Trials ChiCTR1800019349, retrospectively registered on November 7, 2018.

\section{Funding}

The financial support received from different funds was essential to conduct the blood investigations, finance the staff who interviewed the patients, collection of the blood samples, registration and analysis of the data. This work was funded by the National 973 Basic Research Program of China (grant no. 2015CB553604), the Program of Introducing Talents of Discipline to Universities (grant no. B07035), the State Key Program of National Natural Science of China (grant nos. 61331001 and 81530014), and the grants of the Shandong Province Natural Science Fund (grant no. ZR2014CM010). All authors have reported that they have no relationships relevant to the contents of this paper to disclose.

\section{Availability of data and materials}

The datasets used and/or analyzed during the current study are available from the corresponding author on reasonable request.

\section{Authors' contributions}

MN, XX and XL conceived, designed and performed the experiments. MN performed the PCI procedures and extracted coronary blood at the appropriate time. XX, JM, SY and LY collected and analyzed the data. All authors contributed to the interpretation of data, drafted or revised the paper. All authors read and approved the manuscript and agree to be accountable for all aspects of the research in ensuring that the accuracy or integrity of any part of the work are appropriately investigated and resolved.

\section{Ethics approval and consent to participate}

This study was approved by the Regional Ethics Committee of Shandong University Qilu Hospital, China (Reference no. KYLL-2015266). All members of the operation team and patients were informed of the PCI operation, stent implantation, nicorandil injection and coronary blood extraction. Written consent was obtained before PCI and PTCA. This was approved by the Regional Ethics Committee.

\section{Patient consent for publication}

Not applicable.

\section{Competing interests}

The authors declare that they have no competing interests.

\section{References}

1. Ajtay Z, Németh A, Sulyok E, Cziráki A, Szabados S, Martens-Lobenhoffer J, Awiszus F, Szabó C and Bode-Böger SM: Effects of stent implementation on plasma levels of asymmetric dimethylarginine in patients with or without ST-segment elevation acute myocardial infarction. Int J Mol Med 25: 617-624, 2010.

2. Kochiadakis GE, Arfanakis DA, Marketou ME, Skalidis EI, Igoumenidis NE, Nikitovic D, Giaouzaki A, Chlouverakis G and Vardas PE: Oxidative stress changes after stent implantation: A randomized comparative study of sirolimus-eluting and bare metal stents. Int J Cardiol 142: 33-37, 2010.

3. O'Donoghue ML, Morrow DA, Cannon CP, Jarolim P, Desai NR, Sherwood MW, Murphy SA, Gerszten RE and Sabatine MS: Multimarker risk stratification in patients with acute myocardial infarction. J Am Heart Assoc 5: pii: e002586, 2016.

4. Mcclelland RL, Jorgensen NW, Budoff M, Blaha MJ, Post WS, Kronmal RA, Bild DE, Shea S, Liu K, Watson KE, et al: 10-year coronary heart disease risk prediction using coronary artery calcium and traditional risk factors: Derivation in the MESA (Multi-Ethnic Study of Atherosclerosis) with validation in the HNR (Heinz Nixdorf Recall) study and the DHS (Dallas Heart Study). J Am Coll Cardiol 66: 1643-1653, 2015.

5. Liuzzo G, Buffon A, Biasucci LM, Gallimore JR, Caligiuri G, Vitelli A, Altamura S, Ciliberto G, Rebuzzi AG, Crea F, et al: Enhanced inflammatory response to coronary angioplasty in patients with severe unstable angina. Circulation 98: 2370-2376, 1998.

6. KubicaJ,KozinskiM,Krzewina-Kowalska A,Zbikowska-GotzM, Dymek G, Sukiennik A, Piasecki R, Bogdan M, Grzesk G, Chojnicki M, et al: Combined periprocedural evaluation of CRP and TNF-alpha enhances the prediction of clinical restenosis and major adverse cardiac events in patients undergoing percutaneous coronary interventions. Int J Mol Med 16: 173-180, 2005.

7. Buffon A, Liuzzo G, Biasucci LM, Pasqualetti P, Ramazzotti V, Rebuzzi AG, Crea F and Maseri A: Preprocedural serum levels of C-reactive protein predict early complications and late restenosis after coronary angioplasty. J Am Coll Cardiol 34: 1512-1521, 1999.

8. Ikeda U, Ito T and Shimada K: Interleukin-6 and acute coronary syndrome. Clin Cardiol 24: 701-704, 2010.

9. Liuzzo G, Biasucci LM, Gallimore JR, Grillo RL, Rebuzzi AG, Pepys MB and Maseri A: The prognostic value of c-reactive protein and serum amyloid a protein in severe unstable angina. $\mathrm{N}$ Engl J Med 331: 417-424, 1994.

10. Fefer P, Tsimikas S, Segev A, Sparkes J, Otsuma F, Kolodgie F, Virmani R, Juliano J, Charron T and Strauss BH: The role of oxidized phospholipids, lipoprotein (a) and biomarkers of oxidized lipoproteins in chronically occluded coronary arteries in sudden cardiac death and following successful percutaneous revascularization. Cardiovasc Revasc Med 13: 11-19, 2012.

11. Segev A, Strauss BH, Witztum JL, Lau HK and Tsimikas S: Relationship of a comprehensive panel of plasma oxidized low-density lipoprotein markers to angiographic restenosis in patients undergoing percutaneous coronary intervention for stable angina. Am Heart J 150: 1007-1014, 2005. 
12. Tsimikas S, Kiechl S, Willeit J, Mayr M, Miller ER, Kronenberg F, Xu Q, Bergmark C, Weger S, Oberhollenzer F and Witztum JL: Oxidized phospholipids predict the presence and progression of carotid and femoral atherosclerosis and symptomatic cardiovascular disease: Five-year prospective results from the Bruneck study. J Am Coll Cardiol 47: 2219-2228, 2006.

13. Juni RP, Duckers HJ, Vanhoutte PM, Virmani R and Moens AL: Oxidative stress and pathological changes after coronary artery interventions. J Am Coll Cardiol 61: 1471-1481, 2013.

14. Miyazawa A, Ikari Y, Tanabe K, Nakajima H, Aoki J, Iijima R, Nakayama T, Hatori M, Nakazawa G, Tanimoto S, et al: Intracoronary nicorandil prior to reperfusion in acute myocardial infarction. Eurointervention 2: 211-217, 2006.

15. Kim SJ, Kim W, Woo JS, Ha SJ, Kang WY, Hwang SH, Kang DG, Lee SU, Cho SK, Im JS and Kim W: Effect of myocardia protection of intracoronary adenosine and nicorandil injection in patients undergoing non-urgent percutaneous coronary intervention: A randomized controlled trial. Int J Cardiol 158: 88-92, 2012.

16. Chen C, Fu X, Li W, Jia X, Bai S, Geng W, Xing K and Xing K: Intracoronary administration of anisodamine and nicorandil in individuals undergoing primary percutaneous coronary intervention for acute inferior myocardial infarction: A randomized factorial trial. Exp Ther Med 10: 1059-1065, 2015.

17. Ito $\mathrm{H}$, Taniyama $\mathrm{Y}$, Iwakura $\mathrm{K}$, Nishikawa $\mathrm{N}$, Masuyama $\mathrm{T}$, Kuzuya T, Hori M, Higashino Y, Fujii K and Minamino T: Intravenous nicorandil can preserve microvascular integrity and myocardial viability in patients with reperfused anterior wall myocardial infarction. J Am Coll Cardiol 33: 654-660, 1999.

18. Vilalta V, Asmarats L, Ferreira-Neto AN, Maes F, de Freitas Campos Guimarães L, Couture T, Paradis JM, Mohammadi S, Dumont E, Kalavrouziotis D, et al: Incidence, clinical characteristics and impact of acute coronary syndrome following transcatheter aortic valve replacement. JACC Cardiovasc Interv 11: 2523-2533, 2018.

19. Ishii $H$, Ichimiya $S$, Kanashiro $M$, Amano $T$, Imai $K$, Murohara T and Matsubara T: Impact of a single intravenous administration of nicorandil before reperfusion in patients with ST-segment-elevation myocardial infarction. Circulation 112: 1284-1288, 2005.

20. Breuss JM, Cejna M, Bergmeister H, Kadl A, Baumgartl G, Steurer S, Xu Z, Koshelnick Y, Lipp J, De Martin R, et al: Activation of nuclear factor-kappa B significantly contributes to lumen loss in a rabbit iliac artery balloon angioplasty model Circulation 105: 633-638, 2002.

21. van Dijk RA, Kolodgie F, Ravandi A, Leibundgut G, Hu PP, Prasad A, Mahmud E, Dennis E, Curtiss LK, Witztum JL, et al Differential expression of oxidation-specific epitopes and apolipoprotein(a) in progressing and ruptured human coronary and carotid atherosclerotic lesions. J Lipid Res 53: 2773-2790, 2012.

22. Tschoepe D, Schultheiss HP, Kolarov P, Schwippert B, Dannehl K, Nieuwenhuis HK, Kehrel B, Strauer B and Gries FA: Platelet membrane activation markers are predictive for increased risk of acute ischemic events after PTCA. Circulation 88: 37-42, 1993

23. Scholz W and Albus $\mathrm{U}: \mathrm{Na}^{+} / \mathrm{h}^{+}$exchange and its inhibition in cardiac ischemia and reperfusion. Basic Res Cardiol 88: 443-455, 1993.

24. Carreira RS, Monteiro P, Kowaltowski AJ, Gonçalves LM and Providência LA: Nicorandil protects cardiac mitochondria against permeability transition induced by ischemia-reperfusion. J Bioenerg Biomembr 40: 95-102, 2008

25. Zhu F, Zhong X, Zhou Y, Hou Z, Hu H, Liang L, Chen J, Chen Q, Ji $X$ and Shang D: Protective effects of nicorandil against cerebral injury in a swine cardiac arrest model. Exp Ther Med 16: 37-44, 2018.

26. Lee JM, Kato D, Oi M, Toyofuku M, Takashima H, Waseda K, Amano T, Kurita A, Ishihara H, Lim WH, et al: Safety and efficacy of intracoronary nicorandil as hyperaemic agent for invasive physiological assessment: A patient-level pooled analysis. EuroIntervention 12: e208-e215, 2016.

27. Jang HJ, Koo BK, Lee HS, Park JB, Kim JH, Seo MK, Yang HM, Park KW, Nam CW, Doh JH and Kim HS: Safety and efficacy of a novel hyperaemic agent, intracoronary nicorandil, for invasive physiological assessments in the cardiac catheterization laboratory. Eur Heart J 34: 2055-2062, 2013.

28. Kawai Y, Hisamatsu K, Matsubara H, Dan K, Akagi S, Miyaji K, Munemasa M, Fujimoto Y, Kusano KF and Ohe T: Intravenous administration of nicorandil immediately before percutaneous coronary intervention can prevent slow coronary flow phenomenon. Eur Heart J 30: 765-772, 2009.
29. Ota S, Nishikawa H, Takeuchi M, Nakajima K, Nakamura T, Okamoto S, Setsuda M, Makino K, Yamakado T and Nakano T: Impact of nicorandil to prevent reperfusion injury in patients with acute myocardial infarction: Sigmart multicenter angioplasty revascularization trial (SMART). Circ J 70: 1099-1104, 2006.

30. Van KC and Banchereau J: Cd40-cd40 ligand. J Leukocyte Biol 67: 2-17, 2000

31. Henn V, Steinbach S, Büchner K, Presek P and Kroczek RA: The inflammatory action of CD40 ligand (CD154) expressed on activated human platelets is temporally limited by coexpressed CD40. Blood 98: 1047-1054, 2001.

32. Lee Y, Lee WH, Lee SC, Ahn KJ, Choi YH, Park SW, Seo JD and Park JE: CD40L activation in circulating platelets in patients with acute coronary syndrome. Cardiology 92: 11-16, 1999.

33. Mach F, Schönbeck U, Bonnefoy JY, Pober JS and Libby P Activation of monocyte/macrophage functions related to acute atheroma complication by ligation of CD40: Induction of collagenase, stromelysin, and tissue factor. Circulation 96: 396-399, 1997.

34. Cipollone F, Ferri C and Desideri G: Preprocedural level of soluble CD401 is predictive of enhanced inflammatory response and restenosis after coronary angioplasty. Circulation 13: 54-55, 2004.

35. Rondina MT, Lappé JM, Carlquist JF, Muhlestein JB, Kolek MJ, Horne BD, Pearson RR and Anderson JL: Soluble CD40 ligand as a predictor of coronary artery disease and long-term clinical outcomes in stable patients undergoing coronary angiography. Cardiology 109: 196-201, 2008.

36. Dündar C, K1zilırmak F, Tigen K, Izgi A, Karaahmet T, Pala S, Oduncu V, Erkol A, Bulut M and Kırma C: Soluble CD40 ligand release in patients with stable coronary artery disease during elective stent implantation: Effect of drug-eluting stent over bare metal stent. Turk Kardiyol Dern Ars 41: 675-682, 2013.

37. Aströmolsson K, Hedström E, Hultén LM, Wiklund O, Arheden H, Ohlin AK, Gottsäter A and Ohlin H: Dissociation of the inflammatory reaction following PCI for acute myocardial infarction. J Invasive Cardiol 19: 452-456, 2007.

38. Gaspardone A, Crea F, Versaci F, Tomai F, Pellegrino A, Chiariello L and Gioffrè PA: Predictive value of C-reactive protein after successful coronary-artery stenting in patients with stable angina. Am J Cardiol 82: 515-518, 1998

39. Bayata S, Arıkan E, Yesil M, Postacı N, Tas A and Köseoğlu M: An important role for VCAM-1, but not for ICAM-1 in restenosis following coronary stent implantation. Anadolu Kardiyol Der 10: 405-409, 2010

40. Wexberg P, Jordanova N, Strehblow C, Syeda B, Meyer B, Charvat S, Zorn G, Scheinig D, Wojta J, Huber K, et al: Time course of prothrombotic and proinflammatory substance release after intracoronary stent implantation. Thromb Haemost 99: 739-748, 2008

41. Tullio F, Angotti C, Perrelli MG, Penna C and Pagliaro P: Redox balance and cardioprotection. Basic Res Cardiol 108: 392, 2013.

42. Ekeløf S, Jensen SE, Rosenberg J and Gögenur I: Reduced oxidative stress in STEMI patients treated by primary percutaneous coronary intervention and with antioxidant therapy: A systematic review. Cardiovasc Drug Ther 28: 173-181, 2014

43. Demircan S, Yazici M, Diraman E, Demircan G, Kilicaslan F, Durna K, Acar Z and Eren Z: The effect of glucose-insulin-potassium treatment on myocardial oxidative stress in patients with acute coronary syndromes undergoing percutaneous coronary intervention. Coron Artery Dis 19: 99-104, 2008.

44. Li W, Wu N, Shu W, Jia D and Jia P: Pharmacological preconditioning and postconditioning with nicorandil attenuates ischemia/reperfusion-induced myocardial necrosis and apoptosis in hypercholesterolemic rats. Exp Ther Med 10: 2197-2205, 2015.

45. Wang H, Zuo X, Wang Q, Yu Y, Xie L, Wang H, Wu H and Xie W: Nicorandil inhibits hypoxia-induced apoptosis in human pulmonary artery endothelial cells through activation of mitoKATP and regulation of eNOS and the NF- $\mathrm{BB}$ pathway. Int J Mol Med 32: 187-194, 2013.

46. Han Y: Chinese expert consensus on clinical application of ticagrelor. Clin J Med Offic 44: 444-453. 2016.

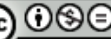

This work is licensed under a Creative Commons Attribution-NonCommercial-NoDerivatives 4.0 International (CC BY-NC-ND 4.0) License. 